\title{
La gestión del posicionamiento analítico de la ciudad como herramienta de marketing urbano: caso ciudad de Matanzas, Cuba
}

\author{
A gestão do posicionamento analítico da cidade como ferramenta do \\ marketing urbano: o caso da cidade de Matanzas, Cuba
}

Managing the analytical positioning of the city as a city marketing tool: the case of Matanzas, in Cuba

Yanlis Rodríguez Veiguela [a] (D), José Armando Pancorbo Sandoval [b], Jensy Tanda Díaz [a], Roberto Carmelo Pons García [a], Sonia Emilia Leyva Ricardo [b]

[a] Universidad de Matanzas, Matanzas, Cuba

[b] Universidad Tecnológica Equinoccial, Santo Domingo, Ecuador

Cómo citar: Veiguela, Y. R., Sandoval, J. A. P,, Díaz, J. T., Carcía, R. C. P. \& Ricardo, S. E. L. (2019). La gestión del posicionamiento analítico de ciudad como herramienta del marketing urbano. Caso ciudad de Matanzas, Cuba. urbe. Revista Brasileira de Gestão Urbana, 11, e20180166. https://doi.org/10.1590/2175-3369.011.e20180166

\section{Resumen}

La creciente rivalidad entre ciudades en el escenario actual marca nuevos retos en la planificación urbana, asumiendo el marketing urbano un rol fundamental, donde aspectos como la competitividad y el posicionamiento se favorecen. Este estudio propone determinar el posicionamiento analítico de una ciudad relacionando aspectos de competitividad y el constructo identidad-comunicación-imagen urbana. Se analizan: (i) la competencia y sus niveles; (ii) su imagen, considerando los índices de valoración e importancia y la correspondencia entre identidad e imagen urbanas; (iii) los atributos identificativos y la posición competitiva. Se utilizaron métodos cualitativo-cuantitativos, destacándose en ellos: la entrevista en profundidad, el método clúster, los índices de valoración e importancia de la imagen urbana, el ideograma e imaograma, la matriz importancia-valoración, y el mapa de posicionamiento. Por este motivo, se identificaron 12 ciudades competidoras de la ciudad de Matanzas, Cuba. Siendo agrupadas en tres niveles de competidores: superior, inferior y semejante. Se evaluó el posicionamiento respecto a los competidores superiores (La Habana, Cienfuegos, Trinidad, Camagüey, y Santiago de Cuba). Se determinaron como atributos identificativos: aspecto físico, patrimonio histórico, y oferta turística. El mapa de posicionamiento evidenció que Matanzas tiene una posición similar a Camagüey y Santiago de Cuba, diferente con Cienfuegos, y opuesta a La Habana y Trinidad.

Palabras clave: Marketing urbano. Competitividad urbana. Planificación estratégica. Posicionamiento de la ciudad.

YRV es Doctora en Ciencias Económicas, Máster en Administración de Empresas - Mención Administración de Negocios, Decana, Presidente Adjunta, e-mail: yanlis.rodriguez@umcc.cu

JAPS es Doctor en Ciencias Económicas y Empresariales, Presidente, e-mail: jose.pancorbo@ute.edu.ec

JTD es Doctora en Ciencias Económicas, Máster en Administración de Empresas - Mención Administración de Negocios, Jefa del

Departamento y docente de Economía, e-mail jensy.tanda@umcc.cu

RCPG es Doctor en Ciencias Económicas y Empresariales, Vicedecano, e-mail: roberto.pons@umcc.cu

SELR es Máster en Contaminación Ambiental - Mención Gestión ambiental y protección de los recursos naturales , profesora,

e-mail: sonia.leyva@ute.edu.ec 


\section{Resumo}

A crescente rivalidade entre cidades no cenário atual marca novos desafios no planejamento urbano. Assim, o marketing urbano assume um papel fundamental, em que aspectos como competitividade e posicionamento são favorecidos. Este estudo propõe determinar o posicionamento analítico de uma cidade relacionando aspectos de competitividade e o construto identidade-comunicação-imagem urbana. Eles analisam: (i) a competição e seus níveis; (ii) sua imagem, considerando os índices de valorização e importância e a correspondência entre identidade e imagem urbanas; (iii) os atributos de identificação e a posição competitiva. Utiliza-se uma metodologia qualitativa-quantitativa, destacando-as: a entrevista em profundidade, o método de cluster, os índices de valoração e importância da imagem urbana, o ideograma e imaograma, a matriz de valorização, e o mapa de posicionamento. Foram utilizados métodos qualitativo-quantitativos, destacando as entrevistas em profundidade, o método de cluster, os índices de avaliação e a importância da imagem urbana, o ideograma e o imaograma, a importância de matriz de avaliação e o mapa de posicionamento. Por esse motivo, foram identificadas 12 cidades concorrentes da cidade de Matanzas, Cuba. Agrupadas em três níveis de competidores: superior, inferior e similar. O posicionamento foi avaliado em relação aos concorrentes superiores (La Habana, Cienfuegos, Trinidad, Camagüey, e Santiago de Cuba). Atributos de identificação foram determinados: aspecto físico, patrimônio histórico, e oferta turística. O mapa de posicionamento mostrou que Matanzas tem uma posição semelhante a Camagüey e Santiago de Cuba, diferente de Cienfuegos, e oposta a Havana e Trinidad.

Palavras-chave: Marketing urbano. Competitividade urbana. Planejamento estratégico. Posicionamento da cidade.

\section{Abstract}

The growing rivalry between cities in the current scenario mark new challenges in urban planning, assigning urban marketing a fundamental role, where aspects such as competitiveness and positioning are favored. This study aimed to determine the analytical positioning of a city relating aspects of competitiveness and the identity-communication-urban image construct. They analyze: (i) the competition and its levels; (ii) its image, considering the valuation and importance indexes and the correspondence between urban identity and image; (iii) its identifying attributes and competitive position. A qualitative-quantitative methodology was used, with particular emphasis on an in-depth interview, the cluster method, the valuation and urban image importance indexes, the ideogram and imaogram, the importance-assessment matrix, and the positioning map. For this reason, 12 cities competing with the city of Matanzas, Cuba, were identified. They were grouped into three levels of competition: superior, inferior and equal. The positioning was evaluated in comparison with the superior competitors (La Habana, Cienfuegos, Trinidad, Camagüey, and Santiago de Cuba). The identification attributes of physical aspect, historical heritage, and tourist offer were determined. The positioning map showed that Matanzas is similar in position to Camagüey and Santiago de Cuba, different from Cienfuegos, and the opposite of Havana and Trinidad.

Keywords: Urban marketing. Urban competitiveness. Strategic planning. Positioning of the city.

\section{Introducción}

La ciudad debe desarrollar una idea creativa construida en torno a atributos tangibles e intangibles; donde las estrategias promovidas ofrezcan trozos de vida, alma y espíritu de las cosas cotidianas de la ciudad, su historia, su arte, su cultura, su deporte, sus negocios, su personalidad y valores, en fin, ofertar su vida plena (Martín, 2004); todo esto puede ser concebido a través de un correcto diseño de la identidad, la imagen y el posicionamiento de ciudad (Rodríguez, 2011; Pancorbo et al., 2016). Determinar el mejor posicionamiento de una ciudad ante sus públicos internos y sus potenciales externos, a través tanto de la mejora del producto (la ciudad en sí misma y sus posibilidades de desarrollo) como a través de la comunicación adecuada y eficaz de la imagen, considerándose que el 
éxito a largo plazo de cada ciudad dependerá del éxito que tengan sus estrategias y de las acciones que desarrolle su competencia para contrarrestarlo (Pancorbo, 1999).

Es por ello que, muchas ciudades van comprendiendo que son poseedoras de atractivos únicos y que los recursos con que cuentan les pueden proporcionar la creación de otros nuevos; siendo necesario que estas identifiquen a sus competidores y analicen en que reside su ventaja competitiva, para el logro del crecimiento y la rentabilidad económica y social, utilizando para ello las técnicas de gestión, en especial el marketing de ciudad, el cual le permitirá el análisis y determinación de dichas ventajas; pues la gran mayoría de estas están asociadas a las características y atributos de la ciudad, los cuales se revelan como un buen instrumento para competir y diferenciarse de otras ciudades a la hora de atraer públicos objetivo o satisfacer a los que ya posee, ya que ciudades aparentemente iguales pueden distinguirse por su identidad e imagen, ofreciendo un valor diferente para quien la percibe.

Estando abocadas a mejorar su gestión a partir de la planificación estratégica del marketing de ciudad, cuyo objetivo fundamental ha de ser el logro del posicionamiento deseado, considerando para ello que estas pueden enfocarse no solo en competir sino también en coopetir; donde aspectos como la identidad y la imagen urbana juegan un papel preponderante, siendo ambas declaradas como objetivos centrales, una vez que la identidad urbana es vista como aquello que caracteriza e identifica a una ciudad y su proyección como imagen urbana, van a aportar los atributos esenciales de la misma, guiándola en su desarrollo estratégico, ya que el sentido de identidad promueve un mayor grado de consenso local y favorece la movilización en torno a los objetivos trazados en los programas de desarrollo, para lograr una mayor identificación de los públicos con el lugar, dotándolo de atributos de identidad específica que las distinga del resto de sus competidoras.

El posicionamiento de ciudad es la definición y el establecimiento de los atributos de identidad a ser proyectados como imagen urbana en lo relativo a la competencia de la ciudad, que le permita la propuesta de acciones de diferenciación en la mente de sus diferentes públicos objetivo para el logro de una posición ventajosa con respecto a la competencia en una categoría determinada.

Coincidiendo con Sanz de la Tajada (1994), Fajardo (2008), Guerrero (2012), Cruz (2013) у Rodríguez (2016) la gestión del posicionamiento consta de tres etapas generales, son:

1. Posicionamiento analítico: determinar exactamente el posicionamiento actual de la ciudad con respecto a las competidoras y en el contexto en el que compiten, consiste en analizar la identidad, examinando sus atributos a proyectar; y la imagen percibida por los públicos objetivo, la imagen de la competencia y los atributos más valorados por el público objetivo al que se dirige, con el fin de conocer cuáles son los atributos que se deben potenciar (Fajardo, 2008; De Elizagarate, 2008; Cruz, 2013; Rodríguez, 2016).

2. Posicionamiento estratégico: resuelto el posicionamiento analítico se podrá abordar el posicionamiento estratégico (Sanz de la Tajada, 1994; Molina, 1995) capaz de determinar cuál es la identidad urbana deseada y la imagen urbana deseada que se quiere obtener (ratificando o rectificando aspectos concretos del posicionamiento analítico) con vista a determinar el posicionamiento deseado de la ciudad, para el logro de este se ha de implementar a partir del desarrollo de estrategias.

3. Control del posicionamiento: la estrategia de posicionamiento es algo vivo, que siempre debe estar presente en la agenda de los directores, y que no es estático, ya que la competencia y las percepciones de los consumidores cambian de forma constante, por lo que el trabajo de posicionamiento ha de ser continuo (Fajardo, 2008) y se debe controlar para poder corregir aquellas estrategias o acciones que no estén acorde con el posicionamiento deseado.

Atendiendo a la importancia que reviste el posicionamiento, en el presente estudio se propone un modelo y un procedimiento para determinar el posicionamiento analítico de una ciudad.

El objeto de estudio será la ciudad de Matanzas, Cuba; considerando que la misma reúne las características siguientes: ser cabecera de la Provincia de Matanzas; con una situación geográfica envidiable que la sitúa entre dos de los polos turísticos (La Habana y Varadero) y en la cercanía de dos 
de los destinos urbanos (Santa Clara y Cienfuegos) más importantes del país; caracterizada por una rica herencia cultural ligada a la identidad nacional y al legado cultural del país donde sobresale el surgimiento del danzón (Baile Nacional de Cuba) y del danzonete así como la creación de la "Bella Cubana" del matancero José S. White pieza emblemática que identifica a Cuba en el mundo; patrimonial, arquitectónica y urbanística siendo la primera ciudad moderna de América, todo ello la llevaron en siglos anteriores a ser reconocida como la "Atenas de Cuba"; con una belleza natural inexplotada a su totalidad y con un potencial endógeno para la inversión y el desarrollo del turismo de ciudad.

\section{Modelo para el posicionamiento analítico de ciudad}

En la literatura consultada tanto a nivel internacional como nacional no se pudieron constatar modelos conceptuales sobre posicionamiento analítico de ciudad. En el caso internacional se constataron algunas metodologías y procedimientos cuyo énfasis recae en la operacionalización de dicha variable. El modelo conceptual será explicado a continuación (Ver Figura 1).

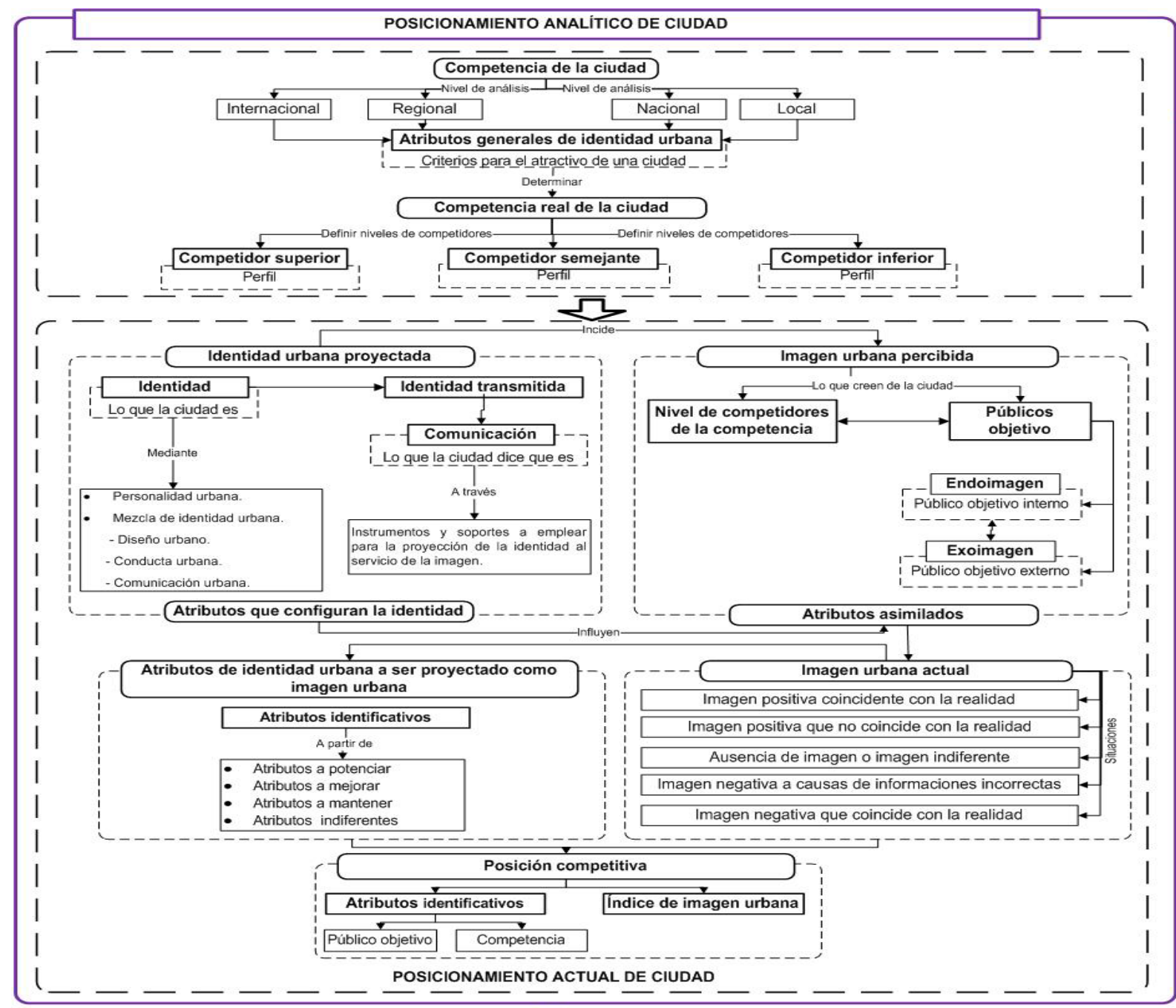

Figura 1 - Esquema del modelo conceptual de posicionamiento analítico de ciudad. Fuente: Rodríguez (2016).

Teniendo en cuenta la concepción de posicionamiento analítico, se hace imprescindible conocer a la competencia y a los públicos objetivo, no todas las ciudades compiten en los mismos segmentos, y lo que es importante realmente es que una ciudad tenga una fuerza relativa mayor en un atributo importante para un grupo meta y que logre diferenciarse con este, así mismo, las ciudades no deben 
corregir necesariamente todas sus debilidades, ni promocionar todas sus fortalezas, sino deben profundizar más en aquellas que afecten más las percepciones y comportamiento del mercado objetivo. De esta forma, aquellas ciudades que deseen ser líderes en un segmento concreto deberán pensar en obtener un atributo valorado por los individuos que los diferencie respecto al resto de las ciudades; proporcionándole más nivel de satisfacción que otros en un aspecto importante para ellos.

Asimismo, los distintos atributos tangibles e intangibles de las ciudades y todos sus activos potencian el valor del conjunto y deben ser desarrollados con el objeto de favorecer el crecimiento de la ciudad y proyectar su imagen a nivel interno y externo. En definitiva, como cualquier producto, las características de la ciudad se revelan como un buen instrumento para competir y diferenciarse de otras ciudades a la hora de atraer clientes o satisfacer a los que ya habitan en ellas.

Especial papel juega en este aspecto el análisis de la imagen percibida a partir de una serie de atributos especialmente seleccionados al respecto, para lo cual en el caso de las ciudades, a nuestro criterio, se debe tener en cuenta el proceso de comunicación de atributos de identidad urbana en términos de imagen con vista al posicionamiento de la ciudad: identidad-comunicación-imagen, el cual se basa en los criterios de Sanz de la Tajada (1994) acerca de las dimensiones de la identidad como eje central para la gestión empresarial, a partir de que las entidades buscan tener una personalidad propia, que permita identificarla, diferenciarla de las demás, dicha personalidad constituye su identidad específica, su propio ser; la identidad de la empresa tiene tres dimensiones conceptuales y operativas, relacionadas en secuencia, las cuales son: lo que la empresa es, lo que ella dice de sí misma que es y lo que los públicos que se relacionan con ella creen que es.

Este es uno de los más completos pues considera las tres dimensiones a su vez y no las concibe como algo aislado y sin correlación, sino con total sinergia entre ellas; a criterio de Carbone (2008) permite incorporar, no solo el ámbito interno de la empresa, sino también, el entorno competidor y los públicos externos, con sus peculiares percepciones en términos de imagen y posicionamiento de la empresa en su propio contexto; ya que los modelos de Costa (1977), Cappriotti (1992), Ind (1992), Van Riel (1997) y Cappriotti (1999) parten del estudio de la imagen de la empresa y los competidores como punto de partida y se centran fundamentalmente en llevar a cabo un análisis de la situación inicial que permita detectar debilidades o puntos fuertes de imagen; mientras que a diferencia de Bernstein (1992) que no hace mención a la imagen de los competidores.

Pons (2000) adapta la propuesta de Sanz de la Tajada (1994) para la gestión de destinos turísticos viéndolo asociado al proceso de comunicación de la imagen como parte del posicionamiento, en la segunda dimensión (comunicación) concibe también la identidad proyectada pero aporta lo referido a la identidad deseada y en la tercera dimensión (imagen) ve la incidencia que tiene la percepción/actitud en la imagen; mientras que Friedmann (2005) lo adapta para la gestión de la ciudad al establecer las siguientes dimensiones de identidad urbana; aspectos con los cuales coinciden Fernández et al. (1997), Molina (2008), Lima (2009), Chaves (2011), Rodríguez (2011), Tanda (2011), González \& Castillo (2011), Guerrero (2012), Cruz (2013) y Cuellar (2014): la dimensión identidad: el ser de la ciudad; la dimensión comunicación: qué hace la ciudad de su propia identidad; la dimensión imagen: es el resultado de la comunicación realizada por la ciudad y percibida en forma de imagen por los distintos públicos, la adecuación fundamental es que lo relaciona con el entorno urbano.

Pero en este caso se adapta la propuesta de Friedmann (2005) con el objetivo de determinar el posicionamiento actual de la ciudad a partir de la imagen urbana actual, teniendo en cuenta que: en la actualidad existe una creciente competencia entre las ciudades, lo cual ha concitado un enorme interés en el concepto de la Identidad Corporativa (Friedmann, 2005); las ciudades no se encuentran ajenas a la incidencia de factores externos que puedan condicionar cambios en las mismas; la identidad es llamada ahora al centro de la cuestión de la competitividad, al ofrecer una vía para la diferenciación de productos (y en consecuencia, para la creación de nichos específicos) en un juego competitivo; la cultura y la identidad socio-territorial que ella produce, aparecen hoy, como factores de competencia y competitividad, al facilitar la construcción y la promoción de imágenes corporativas de ámbito territorial.

Las adaptaciones están enfocadas en que: las ciudades no se encuentran ajenas a la incidencia de factores externos que pueden condicionar cambios en las mismas, como pueden ser la globalización, la migración, entre otros; la existencia de problemas de coherencia entre la identidad proyectada y la imagen percibida, pues las mismas no siempre tienen una relación directamente proporcional y en el caso de la imagen esta depende del público objetivo, por lo que se requiere tener en cuenta la 
diferenciación a partir tanto de la endoimagen como de la exoimagen; igualmente se tiene en cuenta el trabajo con los atributos que configuran la identidad como resultado de la identidad proyectada los cuales influyen en los atributos asimilados a partir de la imagen percibida para cada uno de los públicos objetivo, y los mismos serán usados para poder desarrollar una diferenciación la ciudad teniendo en cuenta para ello que los mismos tienen una estrecha relación con la imagen percibida.

Cuya concepción se encuentra implícita en el modelo conceptual, a partir de que se podrá determinar el posicionamiento analítico de la ciudad, permitiendo el análisis de la imagen de la ciudad de manera tal que resuma y resalte la personalidad de la misma para evitar que pase inadvertida en una sociedad cada vez más globalizada y saturada de estímulos comunicativos, la gestión de esta imagen es una pieza clave para conseguir un posicionamiento deseado (Correa, 2006) a lo cual Paz \& Tkachuk (2004) agregan que es un espejo por medio del cual se reflejen y cristalicen los atractivos de una ciudad.

\section{Procedimiento para determinar el posicionamiento analítico de ciudad}

El objetivo de este procedimiento es determinar el posicionamiento analítico de ciudad con respecto al nivel de competidores, considerando para ello la concepción de la identidad e imagen urbana, lo que permitirá la determinación de los atributos asimilados y su posición competitiva. Se realizará en tres momentos, los cuales son:(Ver Figura 2).

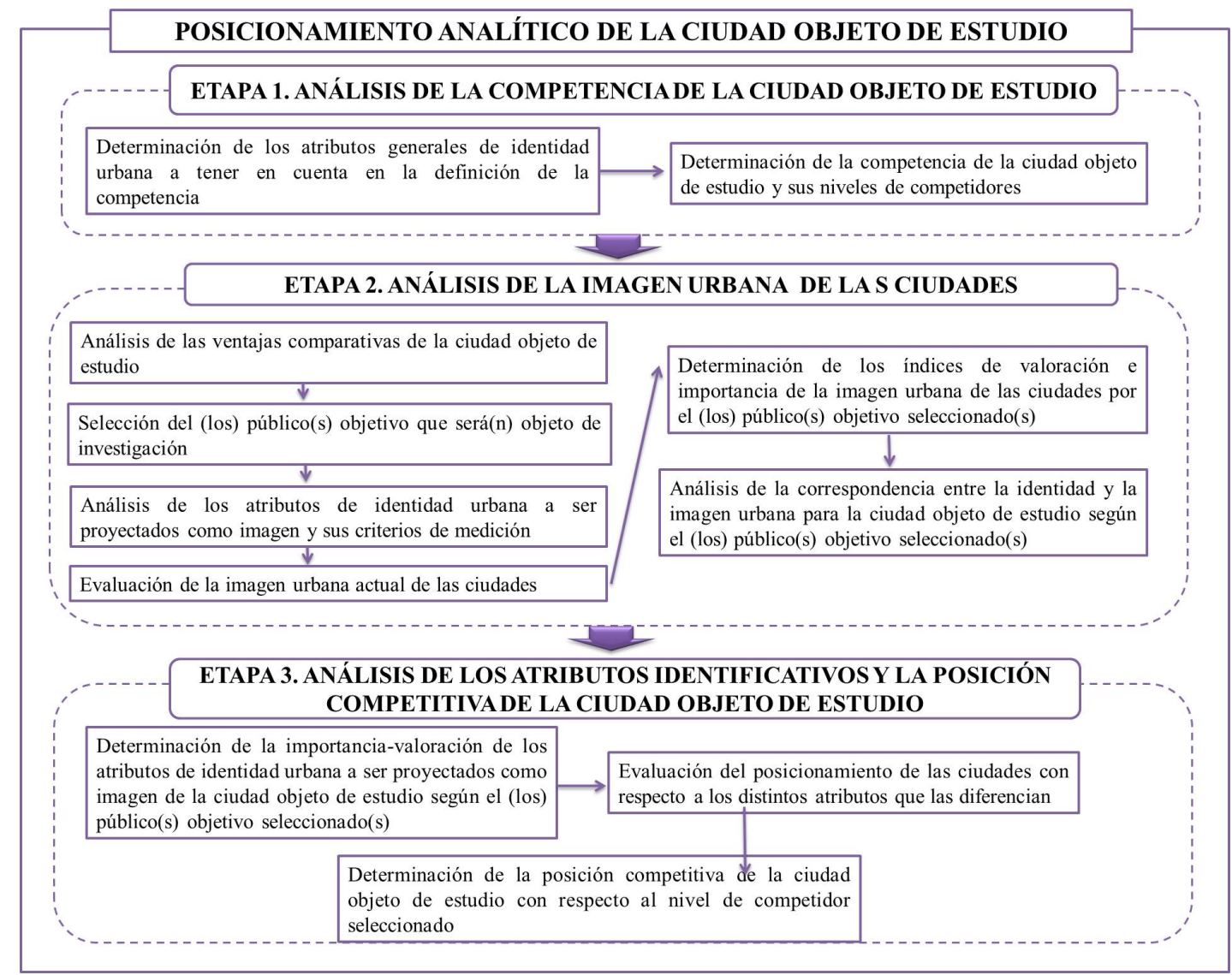

Figura 2 - Esquema del procedimiento para determinar el posicionamiento analítico de la ciudad. Fuente: Rodríguez (2016).

1. Análisis de la competencia de la ciudad objeto de estudio:

Los atributos generales de identidad urbana se determinarán en dos momentos: en el primero a través de una entrevista en profundidad a los expertos considerando para ello los atractivos de las ciudades, los expertos deben ser seleccionados en dependencia del nivel (local, nacional o 
internacional) en que se aplique el procedimiento y su determinación dependerá de sus competencias, considerando para ello el coeficiente K, en este sentido se coincide con Rodríguez (2011) en los pasos para el cálculo del coeficiente de competencia K; posteriormente se debe desarrollar una matriz de síntesis, con el fin de integrar los elementos convergentes planteados por diferentes autores desde el punto de vista teórico. Permitiendo conformar un listado representativo de atributos generales de identidad urbana, donde están concebidos tanto los atributos resultantes de la entrevista en profundidad, como los de la matriz de síntesis.

Para la determinación de la competencia de la ciudad objeto de estudio se realizará a partir de la propuesta de ciudades considerando los atributos determinados anteriormente, mientras que para los niveles de competidores (superiores, semejantes e inferiores) se desarrollará a partir del procedimiento específico para realizar la segmentación de la competencia propuesto por Rodríguez (2016) a partir del método clúster análisis no jerárquico. La elección del nivel de competidor con el cual se debe evaluar el posicionamiento de la ciudad objeto de estudio depende de los intereses de los gestores de la ciudad.

\section{Análisis de la imagen urbana de las ciudades:}

Para el análisis de las ventajas comparativas de la ciudad se deben tener en cuenta fuentes secundarias de información profundizando en la búsqueda de aquellas ventajas comparativas que corresponden a los atributos generales de identidad urbana. En todo proceso de posicionamiento es importante tener en cuenta una selección adecuada del (los) público(s) objetivo.

Posteriormente se debe analizar los atributos de identidad urbana a ser proyectados como imagen, los cuales serán los atributos que se consideren cruciales para la ciudad, seleccionados a partir de los atributos generales de identidad urbana a partir del método Kendall con los expertos seleccionados, en correspondencia con los pasos establecidos por Rodríguez (2011).

Para evaluar la imagen urbana de las ciudades según el o los públicos objetivo seleccionado, se emplean cuestionarios y la estadística básica. El análisis de la valoración y de la importancia de la imagen urbana para cada una de las ciudades, se concreta en la determinación de los índices de valoración e importancia de la imagen urbana de las ciudades aspecto importante para el análisis posterior de la posición competitiva; estos son resultado de la adaptación realizada por Rodríguez (2016) al índice propuesto por Varela et al. (2006) y teniendo en cuenta los criterios de Scheinsohn (1997) y Cappriotti (1999).

El análisis de la correspondencia entre la identidad y la imagen urbana tiene como objetivo establecer las brechas entre la identidad y la imagen urbanas actuales, ya que dichas brechas pueden representar un excedente de imagen sobre identidad, donde la percepción/ evaluación del o los públicos respecto a la misma está por encima de la realidad reflejada en términos de identidad; o puede representar un excedente de identidad o deficiencia de imagen, donde la percepción/ evaluación de la identidad es superior a la imagen percibida actual, lo cual puede estar incidiendo favorable o negativamente en el posicionamiento actual de la ciudad.

Para ello se coincide con Tanda (2011) y Rodríguez (2016) en el procedimiento específico a seguir para realizar este análisis a partir de la representación gráfica de la identidad urbana actual, la que se logra mediante la elaboración del ideograma y en el caso de la imagen urbana actual a partir del imaograma. A partir de las finalidades que persiguen el ideograma y el imaograma, se deduce que ambos deben guardar una estrecha correspondencia, la cual puede ser representada en un iconograma único que contenga al ideograma y al imaograma de la ciudad, y que deje en evidencia gráfica las brechas que puedan existir o si existe correspondencia entre la identidad e imagen urbana actuales, o si hay excedente de imagen o de identidad. Una vez determinadas y graficadas las brechas, se analizaa la identificación de la situación de imagen urbana actual, a partir del criterio de clasificación establecido por Friedmann (1995).

3. Análisis de los atributos identificativos y la posición competitiva de la ciudad: 
La determinación de la importancia-valoración de los atributos de identidad urbana a ser proyectados como imagen de la ciudad objeto de estudio a partir de la matriz importancia-valoración (IPA); criterios que servirán de base para el análisis de estos atributos y su posterior clasificación en atributos a potenciar, mantener, mejorar e indiferentes, los cuales incidirán en la determinación del posicionamiento deseado y una vez concebido este, se estaría en condiciones de poder establecer las prioridades de actuación sobre los mismos, para ser utilizadas por parte de los gestores de la ciudad en la toma de medidas estructurales correspondientes para revertir la situación actual, en el desarrollo de estrategias para el posicionamiento deseado, así como en la mejora de su posición competitiva.

Mientras que en la evaluación del posicionamiento de las ciudades con respecto a los atributos que las diferencian, se definirán qué tan iguales o diferentes las perciben el o los públicos objetivo, lográndose conocer los atributos que la diferencian, los cuales deben utilizados por parte de los gestores de la ciudad para trazar estrategias que permitan lograr la diferenciación con respecto a la competencia y mejorar su posición competitiva.

El análisis de los atributos que diferencian a la ciudad objeto de estudio con respecto al nivel de competidor seleccionado, se tendrán en cuenta a partir del mapa de posicionamiento o perceptual siguiendo el procedimiento de Rodríguez (2016), todo ello facilitaría la toma de decisiones por parte de los gestores locales al conocer las ventajas competitivas de la ciudad objeto de estudio con respecto al nivel de competidor. A partir del análisis de la matriz se puede asumir con cuál de las ciudades dentro del nivel de competidores la ciudad objeto de estudio tiene un posicionamiento similar o diferente, así como cuáles son los atributos que la identifican.

La determinación de la posición competitiva de la ciudad objeto de estudio con respecto al nivel de competidor seleccionado, se debe analizar en dos momentos, el primero, a partir de los resultados de la ciudad objeto de estudio y del nivel de competidores seleccionado, partiendo del índice de valoración de la imagen urbana, logrando ordenar en un orden ascendente las ciudades mejor posicionadas; en un segundo momento, a partir del índice de importancia de la imagen urbana, donde igualmente se ubicaran en un orden ascendente las ciudades, permitiendo conocer a criterio del público objetivo las ciudades que a su criterio tienen más notoriedad para ellos.

\section{Posicionamiento analítico de la ciudad de Matanzas, Cuba}

\section{Análisis de la competencia de la ciudad objeto de estudio}

Los atributos generales que componen la identidad de una ciudad resultante de la entrevista en profundidad con los 18 expertos son: el patrimonio histórico y la relevancia de la arquitectura y urbanismo; el aspecto físico; las ofertas culturales, turísticas y de servicios; la educación y la universidad; el transporte e infraestructura de comunicación; el medio ambiente; la innovación y cultura empresarial; el empleo y la economía; el grado de protección y seguridad, así como las cuestiones sociales; el prestigio y la actitud de la ciudad. Mientras que a partir de la matriz de síntesis, los elementos convergentes, son: arquitectura y urbanismo; patrimonio histórico; oferta cultural; oferta turística; servicios; medio ambiente; transporte e infraestructura de las comunicaciones; actitud de la ciudad; aspecto físico la ciudad y prestigio de la ciudad.

Es importante destacar que existió una marcada coincidencia entre los criterios expuestos por los expertos y los resultados de la matriz de síntesis, lográndose integrar ambos en un listado único de atributos generales de identidad urbana, los cuales son: la educación y la universidad; innovación y cultura empresarial; el empleo y la economía; grado de protección y seguridad; cuestiones sociales; arquitectura y urbanismo; patrimonio histórico; transporte e infraestructura de comunicación; medio ambiente; oferta cultural; oferta turística; servicios; actitud de la ciudad; aspecto físico; prestigio de la ciudad.

En una sesión de trabajo posterior, se llevó a cabo una entrevista en profundidad a los expertos determinándose las ciudades competidoras de Matanzas teniendo en cuenta los atributos generales de identidad urbana; existiendo coincidencia en que son: La Habana, Santa Clara, San Juan de los 
Remedios, Cienfuegos, Sancti Spíritus, Trinidad, Ciego de Ávila, Camagüey, Holguín, Bayamo, Santiago de Cuba y Baracoa.

Posteriormente se procedió al diseño y aplicación del cuestionario para la determinación del nivel de competidores de Matanzas a partir de su competencia, a través del método de ratio, el cual fue validado. A través del análisis clúster, las variables coincidieron con los atributos generales de identidad urbana determinados anteriormente; como medida de similitud, la distancia euclídea cuadrada; mientras que como método clúster, el no jerárquico y el número de entrada para este fue de 3 atendiendo a los niveles de competidores los cuales resultaron ser:

- Conglomerado 1. Competidor superior (La Habana, Cienfuegos, Camagüey, Santiago de Cuba y Trinidad): se caracterizan por evaluaciones altas en la gran mayoría de los atributos solo en los referidos al transporte e infraestructura de comunicación y medio ambiente obtienen resultados regulares, a pesar de ello obtiene las evaluaciones más altas en aspectos. La gran mayoría tienen declaraciones de: Patrimonio Cultural de la Humanidad UNESCO, Patrimonio Mundial, Ciudad Histórica; todas son destino urbano del país y patrimoniales.

- Conglomerado 2. Competidor inferior (Ciego de Ávila y Sancti Spíritus): se caracterizan por evaluaciones bajas en la gran mayoría de los atributos y a pesar de obtener evaluaciones positivas en la arquitectura y urbanismo, la educación y la universidad, el grado de protección y seguridad es el conglomerado que le ofrece valoraciones más bajas. La gran mayoría no tienen declaraciones ni se consideran destino urbano del país y ni patrimoniales, una de ellas si es ciudad monumento nacional; está compuesto por ciudades intermedias.

- Conglomerado 3. Competidor semejante (Santa Clara, Holguín, San Juan de los Remedios, Bayamo y Baracoa): se caracterizan por ser de evaluaciones regulares en la gran mayoría de los atributos, a pesar de que son el segundo grupo que mejor evalúa la oferta turística y el transporte e infraestructura de comunicación, estas son negativas. La gran mayoría tienen declaraciones de: Ciudad Monumento Nacional, son destino urbano del país y patrimoniales; sin embargo no tienen declaraciones de Patrimonio Cultural de la Humanidad" UNESCO, Patrimonio Mundial y Ciudad Histórica; está compuesto por ciudades intermedias.

Teniendo en cuenta los resultados anteriores, la elección del nivel de competidor con el cual se determinará el posicionamiento de la ciudad de Matanzas se realizó a partir del criterio de los actores implicados, los mismos coincidieron que era más oportuno evaluar el posicionamiento de la ciudad de Matanzas con su competidor superior teniendo en cuenta para ello que solo a excepción de Camagüey y Santiago de Cuba, las otras tres ciudades se encuentran en las cercanías de la ciudad; además de considerarse los mejores destinos urbanos del país, criterio que se desea alcanzar para Matanzas en un futuro, en el cual ha incidido el desarrollo de un plan estratégico de ciudad, así como el trabajo que realizan en base de una gestión integrada de ciudad. Igualmente consideran que se está abocando a la diversificación del producto turístico cubano y una muestra de esta estrategia son estas ciudades que más allá de explotar el turismo de sol y playa (convencional) también explotan el turismo urbano, y Matanzas particularmente cuenta con un sin número de potencialidades que hoy no explota con este objetivo, desaprovechando la ventaja de encontrarse solo a 30 minutos de Varadero, el mayor Polo Turístico y destacado balneario del país, además de encontrase en la cercanía de la ciudad de La Habana, destino urbano por excelencia del país, así como se encuentra dentro del corredor turístico de La Habana-Varadero. Asociado a esto se planteó la necesidad del desarrollo local, aspecto que estas ciudades han logrado articular dentro de sus planes estratégicos de ciudad y que igualmente las mismas sobresalen por enfocarse en buscar estructurar sus ofertas de bienes y servicios a sus diferentes públicos objetivo en búsqueda de una imagen más favorable.

\section{Análisis de la imagen urbana de las ciudades}

La ciudad de Matanzas cuenta con atractivos y atributos que la hacen única, muestra de ello son las ventajas comparativas con que cuenta. González etal. (2013) a partir de una revisión exhaustiva de 
documentos, determinaron que la ciudad se caracteriza por un total de 49 primicias, 88 hechos significativos desarrollados en la misma y 33 singularidades que la distinguen de las demás ciudades cubanas.

Considerando la importancia de los públicos objetivo en la actualidad y para el desarrollo de la ciudad de Matanzas, partiendo de la elección del mercado nacional cubano, se seleccionó el público interno cubano y de ellos específicamente los residentes, la muestra quedó definida en 624 residentes después de un muestreo probabilístico.

Como resultado del cálculo del índice de valoración de la imagen urbana: (Ver Tabla 1) se obtuvieron dos grupos bien definidos con respecto a la imagen urbana, el primero compuesto por las ciudades con una imagen más favorable: Trinidad, Cienfuegos y Camagüey, incidiendo en esta situación las evaluaciones positivas de los atributos referidos al patrimonio histórico, el medio ambiente, el aspecto físico, actitud y el prestigio de la ciudad. El segundo grupo estuvo conformado por las ciudades con una imagen menos favorable que son: Matanzas, La Habana y Santiago de Cuba; en este grupo resaltaron las valoraciones negativas otorgadas a los servicios y el medio ambiente de las mismas.

Mientras que como resultado del cálculo del índice de importancia de imagen urbana (Ver Tabla 1), se destaca que todos tuvieron valoraciones muy por encima de las obtenidas en el índice de valoración de la imagen urbana de cada ciudad, lo que denota que el público objetivo considera que estas ciudades tienen un gran potencial por explotar debido a que se sienten motivados por su imagen más allá de su estado actual, además de que estos atributos son prioritarios para las ciudades. En este aspecto se destacó la ciudad de La Habana, a diferencia de la posición rezagada que ocupó con respecto a la valoración que le otorgó el público objetivo, sin embargo esta sí es un reflejo de los valores con que cuenta la ciudad y con los cuales se puede diferenciar y la importancia que revierten para estos.

Tabla 1 - Evaluación de la imagen urbana percibida de las ciudades a partir de los índices

\begin{tabular}{ccc}
\hline Ciudades & $\begin{array}{c}\text { índice de valoración de la imagen } \\
\text { urbana }\end{array}$ & $\begin{array}{c}\text { índice de importancia de la imagen } \\
\text { urbana }\end{array}$ \\
\hline La Habana & 2.6862 & 4.2077 \\
Matanzas & 2.4766 & 4.0917 \\
Cienfuegos & 3.5744 & 3.9553 \\
Trinidad & 3.6396 & 4.3066 \\
Camagüey & 3.5723 & 4.1766 \\
Santiago de Cuba & 3.1145 & 3.9803 \\
\hline
\end{tabular}

Fuente: elaboración propia.

Con respecto a la correspondencia entre la identidad y la imagen urbana, la representación gráfica de la imagen urbana, se consideró oportuno realizar el análisis en estratos, incidiendo en la propuesta de dicha agrupación la presencia en la muestra de unidades muestrales que residen en la ciudad de Matanzas, que van a ver sustentados sus criterios a partir de elementos propios de su identidad, vivencias, expectativas, entre otras, y que condicionarían su percepción de los diferentes atributos y con esto su endoimagen, como es abordado por los especialistas en marketing de ciudades, lo cual podría favorecer la propuesta de estrategias específicas por parte de los gestores de la ciudad para el posicionamiento deseado de Matanzas; y otras unidades muestrales que no, correspondientes al resto de las ciudades seleccionadas, que conformarían lo tratado por los especialistas de marketing de ciudad como exoimagen, que igualmente podría favorecer la propuestas de estrategias generales. Es por ello que quedaron establecidos dos estratos: uno compuesto por los residentes en Matanzas y el otro por los residentes en las otras ciudades, este análisis posibilita una mejor comprensión y análisis de los posibles gaps de imagen que puedan tener cada uno de ellos respecto a la identidad urbana.

En las Figuras 3 y 4 se pueden observar las brechas entre identidad y la imagen urbanas actuales a partir de los distintos iconogramas, conformados por el ideograma y los imaogramas. En ambos casos, se obtuvo una imagen positiva que no coincide con la realidad. 


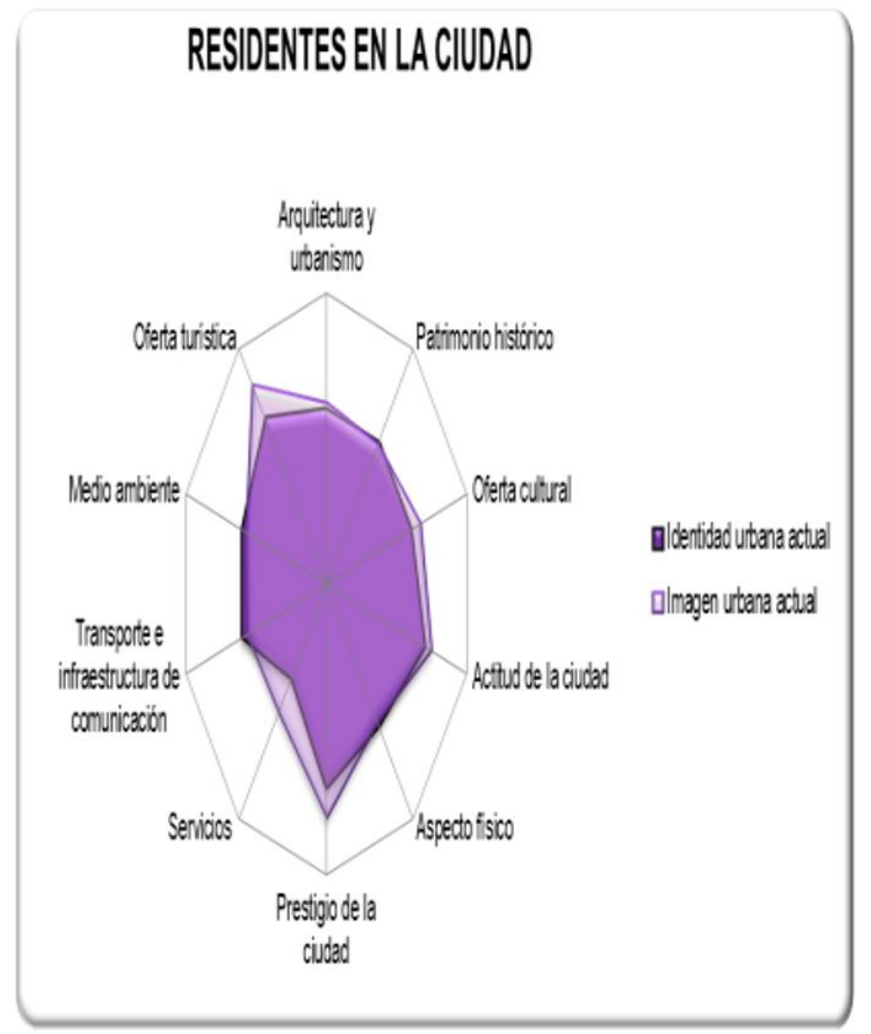

Figura 3 - Iconograma de los residentes en Matanzas. Fuente: elaboración propia.

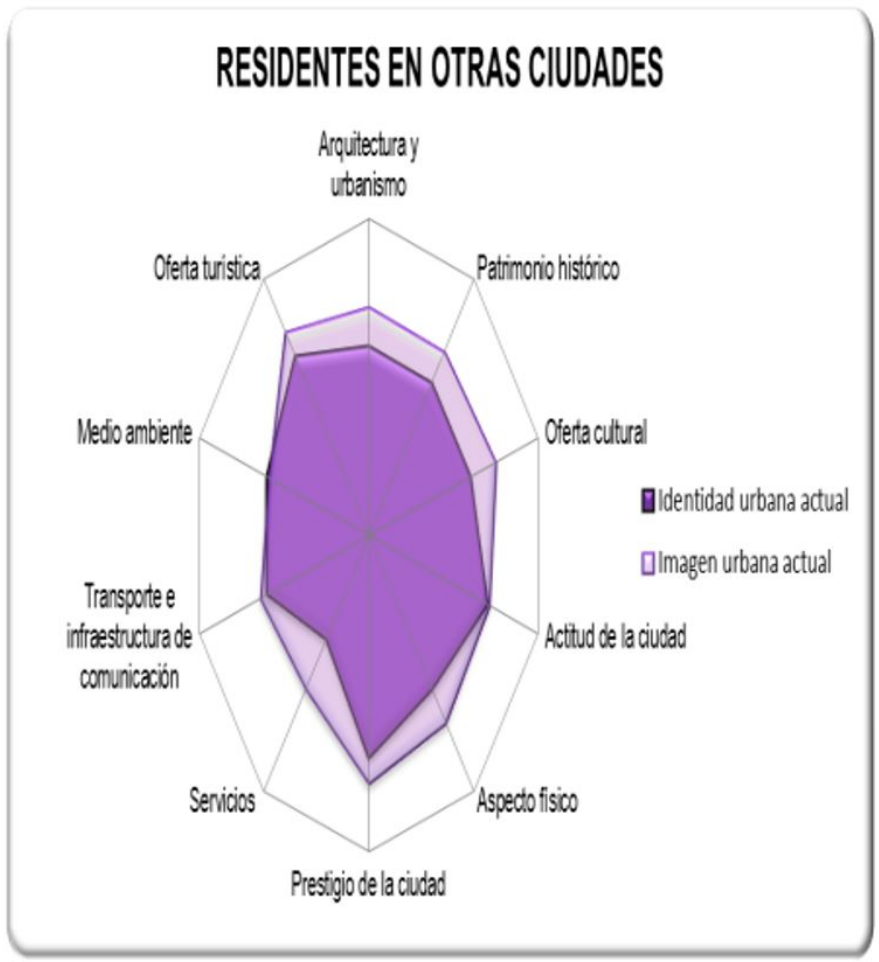

Figura 4 - Iconograma de los residentes otras ciudades. Fuente: elaboración propia.

3. Análisis de los atributos identificativos y la posición competitiva de la ciudad objeto de estudio

Al igual que en los distintos iconogramas, se consideró que es importante tener en cuenta los dos estratos, con el objetivo de poder diferenciar el estado de los atributos, con el fin de que los gestores de la 
ciudad tengan información diferenciada a la hora de desarrollar estrategias para el posicionamiento deseado de Matanzas, que puedan tomar medidas estructurales correspondientes con la realidad para revertir la situación actual y mejorar su posición competitiva. Las matrices importancia-valoración (IPA) para Matanzas (Ver Figura 5 y 6); muestran que los diez atributos analizados en ambos casos están representados por encima de la diagonal del espacio, por lo que todos son susceptibles de mejoras, aunque el nivel de prioridad se establecerá en dependencia de su cercanía o lejanía respecto a la diagonal.

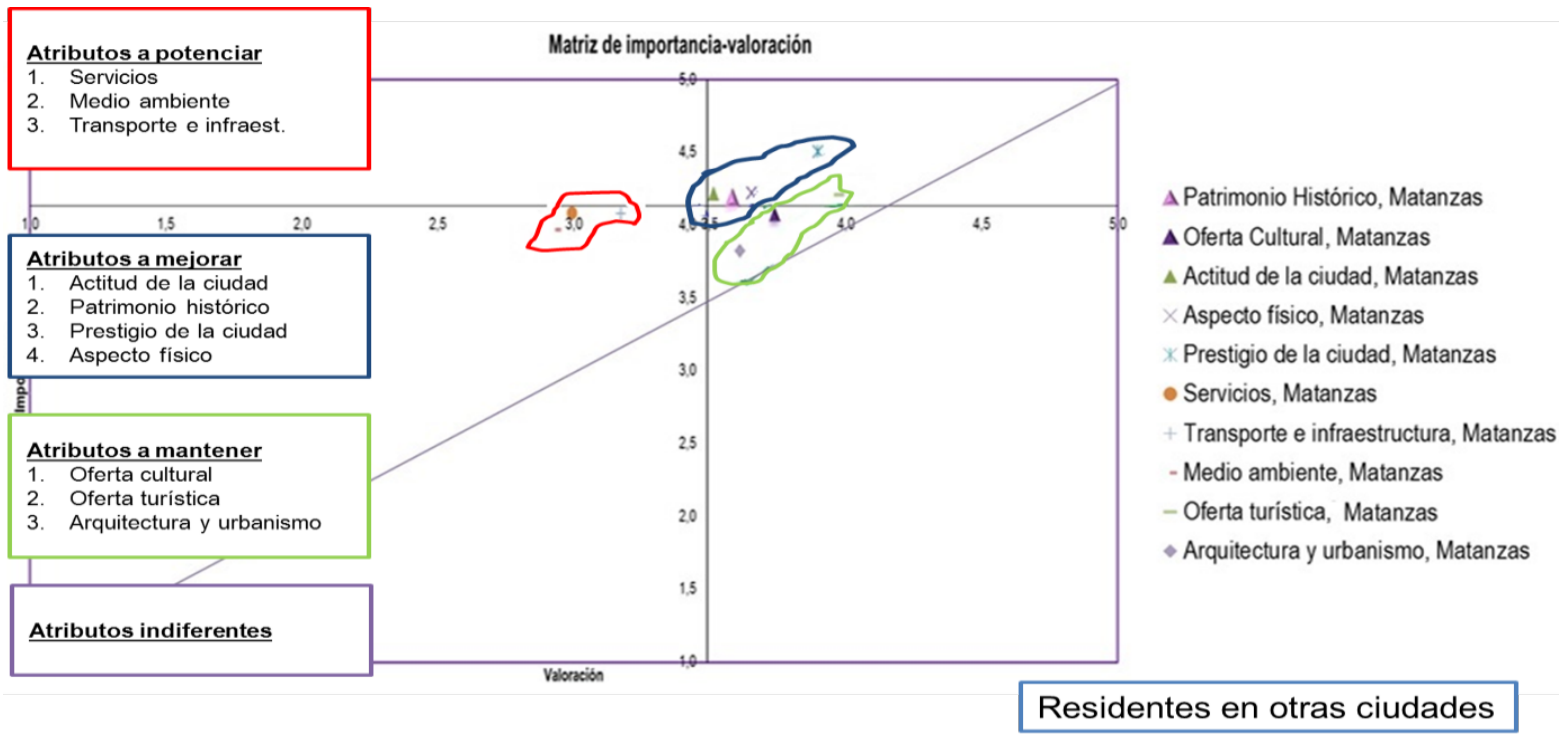

Figura 5 - Matriz IPA de los residentes en otras ciudades. Fuente: Elaboración propia.

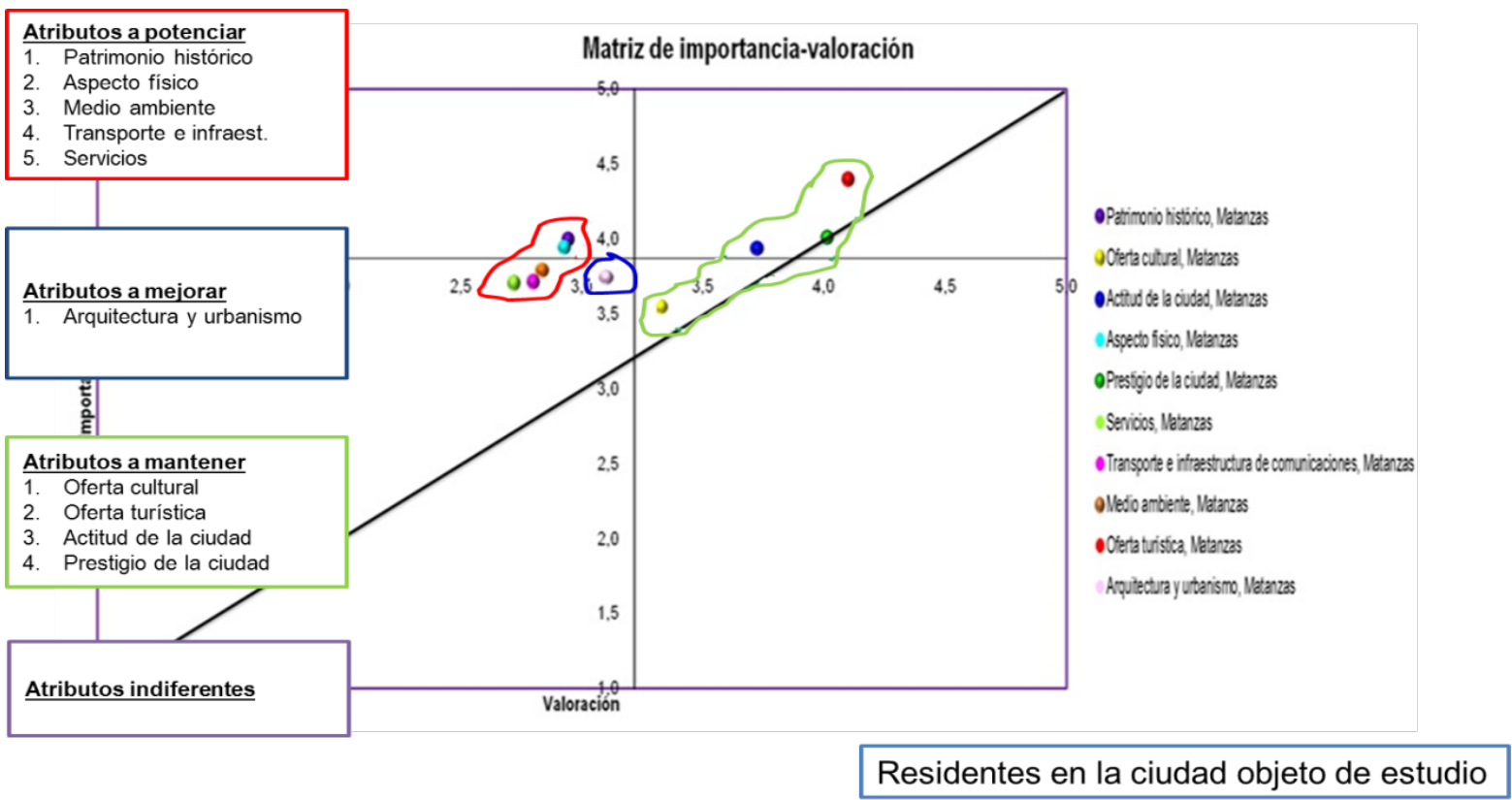

Figura 6 - Matriz IPA de los residentes en Matanzas. Fuente: Elaboración propia.

Grupo 1 (más alejados de la diagonal): atributos que reportaron mayor insatisfacción entre los encuestados en términos de discrepancia. Para encaminar las acciones de mejora, se identificó y estableció la prioridad para su tratamiento, quedando para los residentes en las otras ciudades: servicios, medio ambiente y transporte e infraestructura de comunicación; mientras que para los residentes en Matanzas: patrimonio histórico; aspecto físico; servicios; medio ambiente; transporte e infraestructura de comunicaciones. 
Grupo 2 (intermedios): segundo grupo de atributos con peor satisfacción entre los encuestados en términos de discrepancia. Para encaminar las acciones, se identificó y estableció la prioridad para su tratamiento, quedando para los residentes en las otras ciudades: actitud de la ciudad, patrimonio histórico, prestigio de la ciudad y aspecto físico; mientras que para los residentes en Matanzas: la arquitectura y urbanismo.

Grupo 3 (menos alejados de la diagonal): grupo de atributos de menor discrepancia, es importante destacar que los atributos que conformaron este grupo son en la actualidad desde el punto de vista de los residentes en las otras ciudades los puntos fuertes de la exoimagen de la ciudad de Matanzas, mientras que para los residentes en Matanzas serian de la endoimagen. Para encaminar las acciones, se identificó y estableció la prioridad para su tratamiento, quedando para los residentes en las otras ciudades: oferta cultural, oferta turística y arquitectura y urbanismo; mientras que para los residentes en Matanzas: oferta turística; actitud de la ciudad; oferta cultural; prestigio de la ciudad.

La evaluación del posicionamiento de las ciudades con respecto a los atributos que las diferencian, se realiza teniendo en cuenta el mapa de posicionamiento, el objetivo que se persigue con este es conocer las posiciones relativas de las ciudades entre sí y de éstas respecto a los atributos analizados, para ello se considera que las ciudades son poseedoras de los atributos y por lo tanto son comparables respecto a los mismos; las ciudades son competidoras entre sí. Teniendo en cuenta los resultados del análisis de correspondencia se rechaza la Ho aceptándose la Hi, por lo que si hay ciudades que han recibido una mayor cantidad de asociaciones con un atributo determinado que con otros, siendo una variable dependiente de la otra $(\mathrm{p}<0.05)$. La interpretación del mapa de posicionamiento se realizará en dos momentos: (Ver Figura 7).

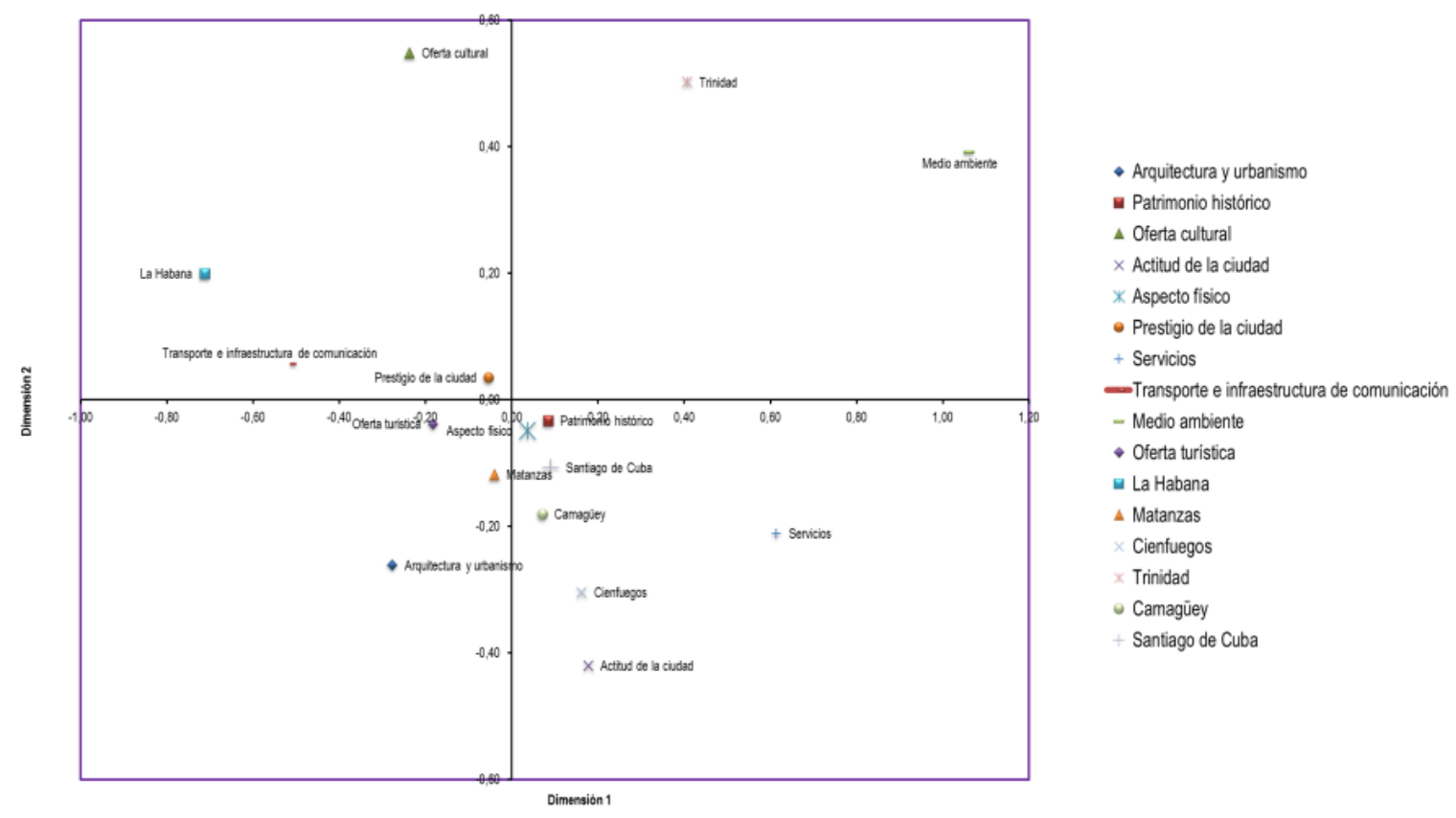

Figura 7 - Mapa de posicionamiento. Fuente: elaboración propia.

4. Relación existente entre la ciudad de Matanzas y las ciudades competidoras, se tiene que:

En un primer momento presentó ángulos más cercanos a $0 \cong$ con Camagüey, Cienfuegos y Santiago de Cuba, cuando se analizó la distancia, la ciudad de Cienfuegos posee una mayor distancia con respecto a Matanzas, por lo que esta posee atributos que la diferencian del resto de las ciudades que conformaban este grupo, es por ello que se puede decir que Camagüey y Santiago de Cuba tienen una correlación positiva muy alta con respecto a Matanzas, las cuales se relacionan por diversos atributos que las caracterizan, en el caso de Cienfuegos existe una correlación positiva pero tiene presencia de 


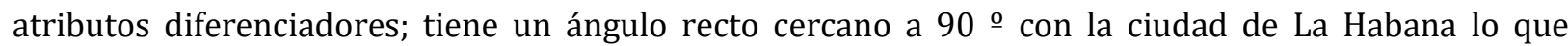
implica muy poca relación entre ellas, además si se tiene en cuenta la distancia esta es superior, logrando atributos identificativos que la diferencia con respecto a Matanzas y generan ventajas competitivas que ninguna otra ciudad posee en la misma medida; en el caso de la relación con la ciudad de Trinidad estas son diametralmente opuestas o lo que es lo mismo hay una fuerte correlación negativa entre ellas. Atendiendo a estos resultados se puede decir que Matanzas tiene una posición similar a Camagüey y Santiago de Cuba, diferente con respecto a Cienfuegos y opuesta con respecto a La Habana y Trinidad.

5. Relación existente entre las ciudades y los atributos, se tiene que:

El público objetivo, a partir del análisis de la correlación y la cercanía de los atributos, estos identifican a: Matanzas con el aspecto físico, el patrimonio histórico y la oferta turística; Camagüey con el aspecto físico, el patrimonio histórico y la oferta turística; Santiago de Cuba con el aspecto físico, el patrimonio histórico y la oferta turística; Cienfuegos con la actitud de la ciudad y la arquitectura y urbanismo; La Habana con el prestigio de la ciudad y el transporte e infraestructura de comunicación; Trinidad con la oferta cultural y el medio ambiente.

Una vez analizado los dos momentos anteriores se puede concluir que existen cuatro grupos de ciudades los que se caracterizan por:

Grupo 1: Santiago de Cuba, Matanzas y Camagüey; las cuales están identificadas por el público objetivo como ciudades que se destacan por atributos como: aspecto físico, el patrimonio histórico y la oferta turística.

Grupo 2: Cienfuegos, que se caracteriza fundamentalmente por su actitud así como por su arquitectura y urbanismo, así esta ciudad forma su imagen en la mente de su público objetivo.

Grupo 3: La Habana, que se caracteriza por ser la ciudad más reconocida por el público objetivo por el estado del transporte y la infraestructura de comunicaciones así como por el prestigio de la misma.

Grupo 4: Trinidad, que se caracteriza por ser la ciudad que se diferencia por su oferta cultural y el medio ambiente.

Para validar las agrupaciones realizadas anteriormente se desarrolló el análisis clúster a partir del método jerárquico o de aglomeración, los datos de entrada que se utilizaron fueron los diez atributos de identidad urbana a ser proyectados como imagen así como las seis ciudades, además de los valores de las puntuaciones de las dimensiones obtenidos en la tarea anterior de este propio paso resultante del análisis de correspondencia.

Como resultado del análisis del dendrograma se pudo constatar que efectivamente son 4 los grupos competitivos de las ciudades y que están identificados con los atributos solo a excepción del medio ambiente el cual no se encuentra identificado con ninguna ciudad en específico, pues se une en el último nivel de aglomeración. Estos grupos a su vez se caracterizan porque las ciudades que los conforman presentan atributos similares entre sí y diferencias notables con respecto a las ciudades de otros grupos.

Con respecto a la posición competitiva que tiene Matanzas con respecto al nivel de competidor superior, se encuentra en una posición competitiva desfavorable a pesar de contar con atributos y atractivos que la hacen única, muestra de ello son las ventajas comparativas y competitivas con que cuenta y que fueron explicadas con antelación. Sin embargo desde el punto de vista de la importancia que le concede el público objetivo a los atributos de la ciudad esta mejora su posición, por lo que si esta se proyecta estratégicamente puede alcanzar dichos valores, por lo que se requiere trabajar en base a una identidad e imagen urbana deseada que permita un posicionamiento deseado.

\section{Conclusiones}

En síntesis el posicionamiento va a permitir resaltar el mejor o los mejores atributos de la ciudad, conocer la posición de los competidores en función a ese o esos atributos, tomar la posición concreta y 
definitiva en la mente del o de los públicos objetivo en perspectiva a los que se dirige una determinada oferta u opción; de manera tal que, frente a una necesidad que dicha oferta u opción pueda satisfacer, los públicos objetivo en perspectiva le den prioridad ante otras similares, permite decidir la estrategia en función de las ventajas y así poder comunicar adecuadamente el posicionamiento al mercado.

Todo lo expuesto anteriormente, permitiría: el desarrollo de intervenciones acertadas por parte de los gestores locales; adecuada planificación de los recursos económicos, financieros y materiales con los que se cuenta; la posibilidad de desarrollar estrategias diferenciadoras a partir de los públicos objetivo y la competencia; diseñar la imagen y crear una deseada por la cual sea reconocida e identificada; evaluaciones y resultados factibles para el desarrollo adecuado del Plan Estratégico de Ciudad, Programa de Identidad Urbana y el Programa de Comunicación de la Ciudad; a partir de aprovechar las ventajas que ofrece para atraer inversiones tanto en el orden empresarial como en las nuevas formas de gestión con el objetivo de crear puestos de trabajos; sedes de organismos nacionales e internacionales; ferias, congresos y exposiciones; visitantes y turistas para el desarrollo del turismo urbano así como residentes; proyectos e inversiones; acontecimientos emblemáticos de tipo deportivo o cultural; infraestructura de conexión y otros; permitiendo ello la elevación de la calidad de vida del ciudadano y de las ofertas en la ciudad.

El posicionamiento actual de la ciudad de Matanzas no es favorable a nivel nacional teniendo en cuenta el criterio del público interno, lo cual se refleja en su posición competitiva; así como por el estado desfavorable de su imagen urbana, muestra de ello son las evaluaciones otorgadas a los atributos seleccionados para el público objetivo.

\section{Referencias}

Bernstein, D. (1992). La imagen de la empresa y la realidad. Crítica de las comunicaciones. Barcelona: Editorial Plaza y Janés.

Carbone, C. (2008). Fundamentos Conceptuales y metodológicos de la imagen de empresa. Introducción a las Relaciones Públicas. Recuperado el 10 de Enero de 2013, de http://www.altillo.com/EXAMENES/uces/publicidad/introrelpublicas/introrelpublicasresumend.asp

Cappriotti, V. P. (1992). La imagen de empresa. Estrategia para comunicación integrada. Barcelona: Editorial El Ateneo.

Cappriotti, V. P. (1999). Planificación estratégica de la imagen. España: Editorial Ariel Comunicación.

Chaves, N. (2011). La gestión de la imagen de la ciudad. Recuperado el 3 de Agosto de 2012, de http://www.creactivistas.com/2011/09/la-gestion-de-la-imagen-de-la-ciudad.html

Correa, E. (2006). Del city marketing al citybranding: construyendo una imagen de ciudad. Recuperado el 8 de Mayo de 2011, de http://www.scielo.cl/scielo.php?pid=s0250-716120000079000004\&scrip=sci-

arttex\&tlng=escomultai

Costa, J. (1977). La imagen de empresa. Métodos de comunicación integral. Madrid: Ibérico-Europea de Ediciones.

Cruz, Y. (2013). Propuesta de niveles de competidores teniendo en cuenta para ello una perspectiva teórica que sustente dicha elección (Tesis de diploma). Universidad de Matanzas, Matanzas.

Cuellar, L.E. (2014). Determinación de la competencia de la ciudad de Matanzas (Tesis de diploma). Universidad de Matanzas, Matanzas.

De Elizagarate, V. (2008). Marketing de ciudades: estrategias para el desarrollo de ciudades atractivas y competitivas en un mundo global. Madrid: Editorial Pirámide.

Fajardo, 0. (2008). El concepto de posicionamiento en las empresas y estrategias para su desarrollo. Recuperado el 10 de Octubre de 2012, de http://fbusiness.wordpress.com/2008/01/05/el-concepto-de-posicionamiento-en-lasempresas-y-estrategias-para-su-desarrollo/ 
Fernández, G., Madoery, O., Gaveglio, S., \& Angelone, J. P. (1997). Posicionamiento, competitividad e imagen de la ciudad de Rosario. Plan estratégico Rosario, posicionamiento, competitividad e imagen de la ciudad de Rosario. Recuperado el 3 de Febrero de 2015, de http://info.worldbank.org/etools/docs/library/112219/peru/docs/ModuloI/Trivelli\%20Rosario\%20Marca\%20 de\%20Ciudad-Lima2003.pdf

Friedmann, R. (1995). Marketing regional: un nuevo instrumento para el desarrollo de las regiones. Revista Chilena de Administración Pública, (7), 1-50.

Friedmann, R. (2005). Marketing estratégico y participativo de ciudades. Recuperado el 9 de Noviembre de 2012, de http: //www.redinternacional.org.mx

González, D., \& Castillo, A. (2011). Procedimiento para posicionar la ciudad de Matanzas a partir de los atributos de identidad urbana a ser proyectados como imagen (Trabajo de diploma). Universidad de Matanzas, Matanzas.

González, L. R., Pérez, L., \& Fernández, D. (2013). Primicias, hechos significativos y singularidades. La Habana: Editorial Universitaria Félix Varela.

Guerrero, E. (2012). Posición competitiva de la ciudad de Matanzas a partir de los atributos de identidad urbana a ser proyectados como imagen (Tesis de diploma). Universidad de Matanzas, Matanzas.

Ind, N. (1992). La imagen corporativa: estrategias para programas de identidad eficaces. Madrid: Editorial Díaz de Santos.

Lima, A. (2009). Estudio de la Imagen Urbana actual de la ciudad Matanzas (Tesis de Diploma). Universidad de Matanzas, Matanzas.

Martín, E. (2004). City marketing. Recuperado el 4 de Abril de 2014, de http://www.futurelx.com/archivos/discurso\%20e_martin.pdf

Molina, A. (2008). Marketing de ciudades. El patrimonio y la cultura local como ejes en la planificación estratégica de la ciudad. Recuperado el 12 de Noviembre de 2011, de http://ubuntuone.com/p/BUi/

Molina, P. (1995). Posicionamiento. La Motorización de los estados mentales de los consumidores. Revista Investigación y Marketing, (49), 45-48.

Pancorbo, J. A. (1999). Desarrollo de indicadores urbanos para la gestión de marketing de ciudad (Tesis doctoral). Universidad de León, España.

Pancorbo, J. A., Anguiano, R., \& Rodríguez, Y. (2016). La marca ciudad como ventaja competitiva para la internacionalización de las ciudades iberoamericanas. Estudio de las ciudades de La Concordia (Ecuador) y Ciudad Obregón (Estado de Sonora, México). Recuperado el 5 de Agosto de 2016, de http://www.clait.com/articulos.htm

Paz, S., \& Tkachuk, C. (2004). Tiempo de city marketing: la imagen de Rosario. In: Anales de lo Seminario Virtual de Imagourbis: Marketing de Ciudades y Desarrollo Urbano (pp. 1-12). Buenos Aires: Universidad Nacional de Quilmes. Recuperado el 16 de Septiembre de 2011, de http://hm.unq.edu.ar/archivos_hm/SP_CT_tiempos_Marketing.pdf

Pons, R. C. (2000). Cuba como destino turístico de "Sol y Playa": imagen y posicionamiento (Tesis doctoral). Universidad de Valencia, España.

Rodríguez, Y. (2011). Valoración de la imagen urbana actual de la ciudad de Matanzas desde la óptica del marketing de ciudad (Tesis de maestría). Universidad de Matanzas, Matanzas.

Rodríguez, Y. (2016). Determinación del posicionamiento deseado en ciudades cubanas: caso ciudad de Matanzas (Tesis doctoral). Universidad de Matanzas, Matanzas.

Sanz de la Tajada, L. A. (1994). Integración de la identidad y la imagen de la empresa. Madrid: ESIC Editorial.

Scheinsohn, D. (1997). Más allá de la imagen corporativa. Cómo crear valor a través de la comunicación estratégica. Buenos Aires: Ediciones Macchi.

Tanda, J. (2011). Fijación de la identidad urbana deseada en la gestión de ciudades: caso ciudad de Matanzas (Tesis doctoral). Universidad de Matanzas, Matanzas. 
Van Riel, C. B. (1997). Principios de comunicación corporativa. Londres: Editorial Prentice-Hall.

Varela, J., García, A., Manzano, V., \& Rial, A. (2006). Development of an index to assess the brand image of tourist destinations. Revista Anales de Psicología, 22(1), 155-160.

Editor: Rodrigo Firmino

Received: Aug. 31, 2018

Approved: Jan. 15, 2019 